Objectives: Describe the clinical characteristics, genetic variants of different autoinflammatory diseases of a cohort of adult patients with follow-up in a 3rd level hospital

Methods: We carried out a descriptive cross-sectional study of adult patients with follow-up in reference hospital consultations with suspicion and/or diagnosis of autoinflammatory disease. Clinical, demographic, and treatment variables were characterised. A descriptive analysis was carried out by subgroup of pathology. The qualitative variables were expressed in frequency and percentages and the quantitative variables in median and interquartile range (IQR). The statistical software IBM SPSS v.18 was used for the analysis

Results: A total of 51 patients were included, 31 women $(60.8 \%)$. Overall, the median age at diagnosis was 28 years (RI 15-40). The most used treatment was colchicine $(60.8 \%)$, steroids $(43.1 \%)$, FAMES $(21.6 \%)$, anti IL-1 $(17.6 \%)$. By subgroup of pathologies the most prevalent with 31 cases $(60.78 \%), 16$ women $(51.6 \%)$ was familial Mediterranean fever (FMF) presenting 15 patients (48.4\%) pathogenic mutations, 8 patients $(25,8 \%)$ low penetrance mutations and the rest $(25.8 \%)$ genetic polymorphisms. The median age at diagnosis was 30.6 years (IQR16-44). The most common symptoms were fatigue $(80.6 \%)$, acute phase reactants (APR) elevation (71\%), fever and myalgias $(67.7 \%)$, arthralgias $(64 \%)$ abdominal pain $(48.4 \%))$, rash $(31 \%)$, arthritis $(29 \%)$, lymphadenopathy $(29 \%)$ serositis $(12.9 \%)$

With regard to TRAPS, 10 patients were diagnosed (19.6\%), 7 women (70\%), with a median age at diagnosis of 26.5 years (IQR 15-31.5). The most common symptoms were arthralgias, fatigue and myalgias (90\%) with elevated APR, fever $(80 \%)$, abdominal pain $(70 \%)$, rash $(60 \%)$ and arthritis $(50 \%)$. There were 2 cases of women (4\%) with Schnitzler syndrome, with arthritis symptoms, elevated APR and chest pain (in 1 case). There were 6 cases $(11.8 \%$ ) of disease associated with NOD 2 gene with a median age at diagnosis of 30 years (IQR 12.5-40), 4 were women $(66.7 \%)$. The most common symptoms were arthralgias, fever and myalgias (83.3\%) with increased APR, arthritis (66.7\%), abdominal pain, oral thrush and rash $(66.7 \%)$.

In the disease associated with NLRP12 gene there were 2 cases $(3.9 \%)$, both cases were women. The most common symptoms were arthralgias, arthritis, fever, fatigue, increased APR and serositis (in one case). There was a case of sickness associated with the NLRP3 gene (cryopyrinopathy) (2\%) in a woman with fever and refractory pyoderma gangrenosum and another woman with a diagnosis of Muckle-Wells (2\%) with clinical signs of deafness since childhood, conjunctivitis, fever and arthritis. There was a case of PFAPA (2\%) in a woman with fever, pharyngitis, rash and lymphadenopathy.

Conclusions: In our series, the most frequent pathology was FMF followed by TRAPS. The most prevalent symptoms were systemic manifestations (fever, fatigue, APR elevation) and musculoskeletal manifestations.

Disclosure of Interest: None declared

DOI: 10.1136/annrheumdis-2018-eular.7434

\section{THU0608 \\ THE RELATIONSHIP BETWEEN SERUM SOLUBLE KLOTHO, FGF-23 LEVELS AND FLOW-MEDIATED DILATATION (FMD) IN PATIENTS WITH FAMILIAL MEDITERRANEAN FEVER (FMF)}

M. Celik ${ }^{1}$, F. Candan ${ }^{2}$, M.E. Derin ${ }^{3}$, I. Karadağ ${ }^{3}$, M. Timuçin ${ }^{2}$, H.O. Doğan ${ }^{4}$, A C. Urhan ${ }^{3}$, M. Şahin ${ }^{4}$, A. Şahin ${ }^{3} .{ }^{1}$ Internal Medicine; ${ }^{2}$ Internal Medicine Nephrology; ${ }^{3}$ Rheumatology - Internal Medicine; ${ }^{4}$ Biochemistry, Cumhuriyet University Medical Faculty, Sivas, Turkey

Background: Familial Mediterranean fever (FMF) is a disease having inflammatory attacks. ${ }^{1}$ Systemic inflammation has an important role in the etiology of endothelial dysfunction. Fibroblast Growth Factor 23 (FGF-23) is expressed by osteoblasts and needs serum soluble klotho. FGF-23 provides phosphate regulation. Increased FGF-23 is associated endothelial dysfunction. ${ }^{2}$ An invasive way of measuring endothelial dysfunction is Flow-Mediated dilatation (FMD). ${ }^{3}$

Objectives: To investigate the possible relationship between FGF23, serum soluble klotho levels and FMD in patients with FMF and healthy subjects.

Methods: Between March 2017 - September 2017, sixty -FMF patients that following-up at Cumhuriyet University Medical Faculty Rheumatology-Internal Medicine Department and thirty healthy volunteers were included into the study. Blood samples were taken from all participants and serum soluble klotho, FGF23 values were measured. Clinical findings of all patients were recorded. Blood tests were examined by Elisa method in Cumhuriyet University Department of Biochemistry. FMD assessed by Doppler ultrasound in all participants.

Results: Mean serum FGF 23 level was measured as $221.01 \mathrm{pg} / \mathrm{ml} \mathrm{pg}$ in FMF group and $99.4 \mathrm{pg} / \mathrm{ml}$ in healthy control $(\mathrm{HC})$ group. There was statistically significant difference between two groups $(\mathrm{p}<0.05)$. The mean serum levels of serum soluble Klotho was measured as $3.0 \mathrm{pg} / \mathrm{ml} \mathrm{FMF} \mathrm{group} \mathrm{and} 8.25 \mathrm{pg} / \mathrm{ml}$ in the $\mathrm{HC}$ group. There was statistically significant difference between the two groups $(p<0.05)$ (figure 1). The mean percentage of FMD was measured as 10.1 in FMF group and 13.6 in the $\mathrm{HC}$ group. There was statistically significant difference between the two groups $(p<0.05)$.

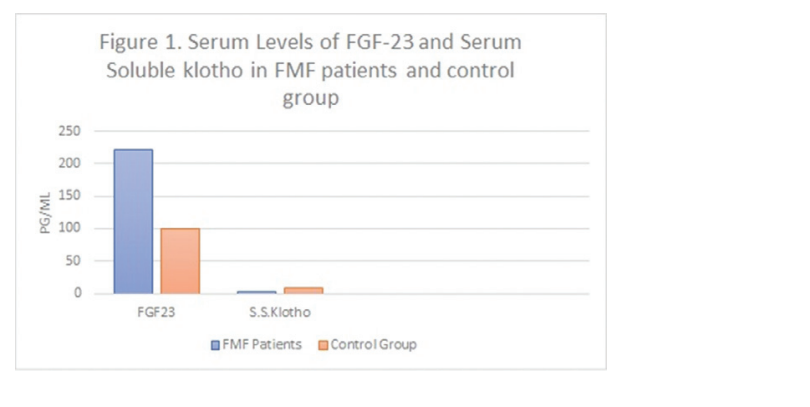

Abstract THU0608 - Figure 1 Serum. Levels of FGF-23 and Serum Soluble klotho in

FMF patients and control group

Conclusions: We found that high FGF 23 levels in FMF patients caused a significant decrease in FMD. In addition, FGF23 may be a parameter for the early diag nosis and prognosis of possible cardiovascular events showing significant change in FMD which demonstrates endothelial dysfunction indirectly.

\section{REFERENCES}

[1] Sahin A, Karakus S, Durmaz Y, et al. Evaluation of Ovarian Reserve with Anti-Müllerian Hormone in Familial Mediterranean Fever. Int $\mathrm{J}$ Rheumatol 2015;2015:380354. doi:10.1155/2015/380354. Epub 2015 May 12

[2] Silswal N, Touchberry CD, Daniel DR, et al. FGF23 directly impairs endo thelium-dependent vasorelaxation by increasing superoxide levels and reducing nitric oxide bioavailability. Am J Physiol Endocrinol Metab 2014 Sep 1;307(5)

[3] Ghiadoni L, Salvetti M, Muiesan ML, Taddei S. Evaluation of endothelia function by flow mediated dilation: methodological issues and clinical importance. High Blood Press Cardiovasc Prev 2015 Mar;22(1):17-22.

Acknowledgements: We would like to thank to "Cumhuriyet University Scientifical Projects Unit (CUBAP)" for funding this project.

Disclosure of Interest: None declared

DOI: 10.1136/annrheumdis-2018-eular.4020

\section{THU0609 CLINICAL CHARACTERISTICS AND OUTCOME OF SPANISH PATIENTS WITH ACUTE MYELITIS ASSOCIATED WITH AUTOIMMUNE DISEASES}

A. González García ${ }^{1}$, W.A. Sifuentes Giraldo² ${ }^{2}$ S. Sainz de la Maza ${ }^{3}$, I. Barbolla Díaz ${ }^{1}$, J. L ${ }^{1}$, L. Manzano Espinosa ${ }^{1} .{ }^{1}$ Internal Medicine; ${ }^{2}$ Rheumatology;

${ }^{3}$ Neurology, Hospital Ramón y Cajal, Madrid, Spain

Background: Acute myelitis (AM) is a focal inflammatory disorder of the spinal cord characterised by motor, sensory, and autonomic dysfunctions that usually develop during a short period (several hours to days) and may resolve over several weeks to months. Acute has been reported as an unusual complication of autoimmune diseases (AD), mainly in systemic lupus erythematosus (SLE) and Sjögren's syndrome (SS).

Objectives: To analyse the frequency of concomitant AD in patients with patients with AM seen in a Spanish tertiary centre and to compare their clinical characteristics and outcome with those of AM patients without other AD.

Methods: We performed a retrospective study including all the patients diagnosed with AM in our centre between January 1989 and December 2017. Patients with previous history of spinal disease (multiple sclerosis [MS], compression, trauma, arteriovenous malformation, radiotherapy) and children were excluded. Demographics, clinical, laboratory, imagenology, therapeutic and outcome data were obtained from their medical records.

Results: During the study period 144 cases of AM were identified, 76 of them had complete data and were analysed. Most of the patient were women (47,62\%), with a mean age at diagnosis $42 \pm 17$ years. The main causes of $A M$ in our series were MS (35\%), AD (18\%), postviral myelitis (9\%) and idiopathic (34\%). The AD diagnosed in these patients were: SLE $,{ }^{4} \mathrm{SS},{ }^{3}$ undifferentiated connective tissue disease ${ }^{2}$ Graves' disease, ${ }^{1}$ ulcerative colitis, ${ }^{1}$ polymyalgia rheumatic, ${ }^{1}$ cryoglobulinemic vasculitis ${ }^{1}$ and sarcoidosis. ${ }^{1}$ A patient with MS had concomitantly another $A D$ (inflammatory myopathy). Most of the patients with $A D(12,80 \%)$ were diagnosed of these disorders before the AM episode (median 48 months, range: $24-120$ ). No differences regarding to sex, age and anatomical extension of $A M$ were detected between $A D$ and non- $A D$ patients. The recurrence of $A M$ was more frequent in non-AD patients $(7 \%$ vs $54 \%, p<0.001)$ and a tendency to less severe clinical course was found in AD patients ( $67 \%$ vs $41 \%, p=0.08$ ).

Conclusions: $A D$ were an important cause of $A M$ in our series and this usually occurred after diagnose of $A D$, so it is important to consider $A M$ when these patients develop neurological dysfunctions that suggest spinal cord damage. The outcome of $A M$ seem to be better in patients with concomitant $A D$. 